\title{
Uso de próteses removíveis em pacientes com necessidades especiais: risco associado ao diagnóstico
}

\author{
- Patricia Kapicius Departamento de Patologia Oral e Maxilofacial, Faculdade de Odontologia, Universidade de São Paulo, \\ São Paulo, SP, Brasil • Fabio Daumas Nunes Disciplina de Patologia Bucal, Faculdade de Odontologia, Universidade de \\ São Paulo, São Paulo, SP, Brasil • Marcel Lautenschlager Arriaga Faculdade de Odontologia, Universidade Federal da \\ Bahia, Salvador, BA, Brasil • Ana Lúcia Aparecida Bronzo de Freitas Centro de Especialidades Odontológicas da Estância \\ Hidromineral de Poá, Poá, SP, Brasil • João Jurandir Simões Júnior Centro de Especialidades Odontológicas da Estância \\ Hidromineral de Poá, Poá, SP, Brasil • Regiana de Souza Carlini Secretaria da Saúde da Estância Hidromineral de Poá, Poá, \\ SP, Brasil
}

RESUMO | Objetivos: Avaliar variáveis associadas à necessidade do uso de prótese dos pacientes com necessidades especiais (PNE) do Centro de Especialidades Odontológicas da Estância Hidromineral de Poá (CEO-Poá). Materiais e métodos: A amostra total foi composta por 514 PNE do CEO-Poá, e a variável dependente foi a necessidade de uso de prótese estabelecida entre janeiro de 2011 e dezembro de 2016. As variáveis foram gênero, idade, diagnóstico principal, quantidade de medicação utilizada usualmente e aspectos comportamentais e foram compiladas do prontuário físico para o formulário eletrônico desenvolvido para este estudo. Dados foram tabulados e analisados. Resultados: Foram encaminhados 88 indivíduos (17,11\%) à reabilitação protética. Prevaleceu o gênero feminino e o avanço da idade aumentou a necessidade de uso de prótese, de forma semelhante à população geral. O diagnóstico principal foi uma variável influenciadora, a maioria (47,72\%) apresentou alterações sistêmicas, seguido de deficiência física (18,18\%) e distúrbios psiquiátricos (11,36\%). Quanto ao comportamento, todos indivíduos foram atendidos em âmbito ambulatorial, a maioria (71,59\%) já havia recebido anestesia em tratamentos anteriores, e apenas 7,95\% precisou de condicionamento psicológico. Grande parte dos PNE (89,77\%) chegou a receber e utilizar as próteses. Conclusões: O diagnóstico principal e variáveis do perfil comportamental foram influenciadores na necessidade de próteses dos PNE do CEO-Poá. Gênero e idade igualmente influenciam a indicação, assim como na população em geral. Esse estudo poderá nortear a implementação de projetos tanto no setor privado como no público.

DESCRITORES | Próteses; Diagnósticos; Doenças; Pacientes com Necessidades Especiais; Tratamento Odontológico; Odontologia.

ABSTRACT | Removable prostheses in patients with special needs: risk associated with diagnosis - Objectives: To evaluate variables associated with the need of denture by patients with special needs attending the Dental Specialty Center (DSC) in Poá, São Paulo, Brazil. Materials and methods: The total sample consisted of 514 patients, treated at the DSC-Poá, and the dependent variable was need of denture established between January 2011 and December 2016. The analyzed variables were gender, age, main diagnosis, amount of medication used, and behavioral aspects. The physical records were compiled to an electronic form specially developed for this study. Data were tabulated and analyzed. Results: Eighty-eight patients (17.11\%) were referred to prosthetic rehabilitation. The female gender prevailed and the need for denture increased with age, in a similar manner as in the general population. Main diagnosis was a relevant variable, with the majority (47.72\%) presenting systemic alterations, followed by physical deficiency (18.18\%) and psychiatric disorders (11.36\%). Regarding the behavior, all individuals were attended as outpatients, the majority (71.59\%) had already received anesthesia in previous treatments, and only 7.95\% required psychological conditioning. Most of the patients with special needs (89.77\%) came to receive and use the prostheses. Conclusions: Main diagnosis and behavioral profile variables influenced the denture indication for those patients at the DSC-Poá. Gender and age also influence indication, as seen in the general population. This study may guide the implementation of projects both in private and public sectors.

DESCRIPTORS | Prostheses; Diagnosis; Diseases; Special Needs Care; Dental Treatment; Odontology.

AUTOR CORRESPONDENTE | - Maria Patricia Kapicius Mariano Caires Departamento de Patologia Oral e Maxilofacial, Faculdade de Odontologia, Universidade de São Paulo • Rua Cel. Cardoso de Siqueira, 3232, CP 240 Mogi das Cruzes, SP, Brasil • 08790-420 E-mail: patricia.kapicius.caires@gmail.com

- Received Dec 4,2017 • Accepted Jan 11, 2018

- Dol http://dx.doi.org/10.11606/issn.2357-8041.clrd.2018.141228 


\section{INTRODUÇÃO}

A qualidade de vida relacionada à saúde (QVRS) é prioridade na comunidade científica. Na odontologia não é diferente; estudos apontam que condições bucais desfavoráveis, como dentes ausentes, estão negativamente associadas ao bemestar da população em geral ${ }^{1}$. O edentulismo é um problema multifatorial que inclui falhas na higiene bucal, traumatismos e estado de saúde debilitado, e compromete aspectos sociais, comportamentais, psicológicos e funcionais ${ }^{2}$.

As perdas dentárias são mais impactantes ainda em pacientes com necessidades especiais (PNE), pois esse grupo apresenta peculiaridades no atendimento - no qual a experiência e conhecimentos específicos dos cirurgiões-dentistas são primordiais ${ }^{3}$. Por isso foi oficializada a especialidade Odontologia para PNE, cujo objetivo é o diagnóstico, a prevenção, o tratamento e o controle dos problemas de saúde bucal das pessoas que apresentam maior complexidade biológica e/ou psicológica e/ou social, bem como necessidade de atuação transdisciplinar com outros profissionais de saúde e de áreas correlatas ${ }^{4}$. Além disso, trata-se de uma população que inclui doenças diversas e que pode exigir colaboração mais intensa dos familiares, na dependência do estado geral de saúde e da medicação utilizada pelo paciente ${ }^{5}$.

Alguns sinais relatados em diversas doenças, como rigidez muscular, tremores, movimentos involuntários de língua e lábios, podem inviabilizar o tratamento do edentulismo por próteses removíveis, tanto as totais como as parciais ${ }^{6}$. O manejo difícil nas etapas de execução podem ter como consequência falta de retenção, dificuldades no ajuste, inflamação da mucosa oral e estética inadequada nas próteses, podendo não serem aceitas dependendo do indivíduo. Adicionalmente, nas próteses removíveis parciais podem ocorrer fraturas dos grampos da armação ${ }^{7,8}$.

O objetivo deste estudo é descrever o perfil dos PNE do Centro de Especialidades Odontológicas da Estância Hidromineral de Poá (CEO-Poá) que necessitaram de próteses totais ou removíveis, e identificar as variáveis influenciadoras. Portanto, as informações epidemiológicas apresentadas neste estudo são importantes, pois podem propiciar melhor compreensão da população estudada e difundir cientificamente critérios de reabilitação oral, de implementação e otimização de serviços públicos de prótese. O desenvolvimento de estratégias para melhoria da saúde bucal tem influência direta na saúde física e mental, sendo fundamental na qualidade de vida dos pacientes com necessidades especiais.

\section{MATERIAIS E MÉTODOS}

Este estudo foi observacional descritivo retrospectivo, com amostra $(n=514)$ que incluía indivíduos atendidos pelas CD especialistas em PNE no período de janeiro de 2011 a dezembro de 2016, no CEO-Poá, situado no estado de São Paulo, Brasil. Desse total de pacientes, aqueles edentados que estavam aptos para manejo, moldagens, aceitação e adaptação às próteses removíveis foram 88 .

Como o objetivo do estudo foi observar fatores comportamentais e de perfil que influenciassem na necessidade de próteses pelos PNE, não foram discriminadas próteses totais de removíveis. Para definição do perfil foram considerados o gênero, a idade e o diagnóstico principal, segundo a classificação discutida na II Assembleia Nacional de Especialidades Odontológicas, em 2001, e na reunião da Associação Brasileira de Odontologia para Pacientes Especiais de 20029. Quanto ao comportamento e à história médica, os fatores analisados foram: âmbito do atendimento, necessidade de condicionamento, finalização do tratamento odontológico, aceitação de anestesia local em tratamento anterior, e quantidade de medicações utilizadas usualmente por esses indivíduos.

O termo de esclarecimento foi assinado pelo paciente ou responsável, que também apresentou laudos médicos comprovando dados. Duas especialistas em PNE padronizaram anamnese, 
exames clínicos, preenchimento dos prontuários, planejamentos e atendimento odontológico durante o período estudado. Os dados constantes nos prontuários foram transferidos por uma das pesquisadoras para formulário eletrônico, otimizado para esse estudo utilizando o software Epi Info versão 7.0. A relação entre as variáveis só foi considerada estatisticamente significante para valores com p menor que 0,05.

O Comitê de Ética e Pesquisa da Universidade de São Paulo aprovou esta pesquisa sob o número 71943617.8.0000.0075, e a instituição estudada autorizou o uso dos dados por escrito.

\section{RESULTADOS}

A amostra $(\mathrm{n}=514)$ incluiu todos os PNE atendidos no CEO-Poá, sendo 264 do gênero masculino $(51,28 \%)$, e a maioria portadores de alterações sistêmicas (32,30\%), seguido de deficiências físicas $(16,54 \%)$ e mentais $(12,06 \%)$. Apresentaram baixa prevalência de necessidade de prótese, com apenas 88 indivíduos (17,12\%).

Quanto a esses PNE encaminhados à reabilitação oral, prevaleceu o gênero feminino (60,47\%), com média de 56 anos, sendo a maioria com alterações sistêmicas, seguida de deficiências físicas e distúrbios psiquiátricos.

Uma variável influenciadora foi a idade, que aumentou em proporção direta à necessidade do uso de próteses (Figura 1). A maioria, 30 casos (34,09\%), estava entre 60 e 69 anos, seguida de 22 indivíduos (25\%) entre 50 e 59 anos. A minoria era formada por jovens entre 20 e 29 anos, com apenas 2 indicações $(2,27 \%)$.

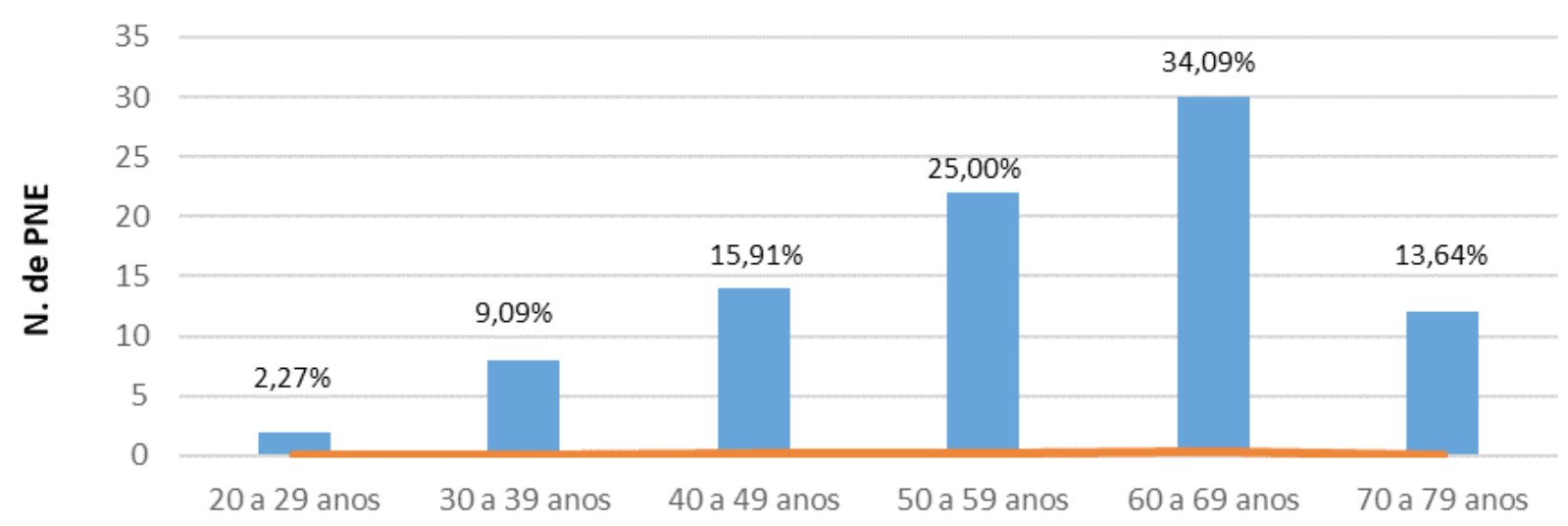

Faixa etária dos PNE que necessitaram do uso de prótese

Figura 1 | Distribuição dos PNE que necessitaram de próteses, segundo faixa etária.

Os 88 pacientes analisados foram classificados em dez grupos de PNE: deficiência mental; deficiência física; anomalias congênitas; autismo; distúrbios psiquiátricos; alterações sensoriais; alterações sistêmicas; doenças infectocontagiosas; condições sistêmicas; e diagnóstico médico indefinido (Tabela 1). O maior grupo de pacientes $(25,30 \%)$ consistia de indivíduos com alterações sistêmicas, como hipertensão arterial sistêmica, diabetes mellitus e cardiopatias.

Já entre os pacientes com distúrbios psiquiátricos, 10 (22,22\%) deles necessitaram de prótese. Entretanto, os deficientes físicos, devido às sequelas de AVC, poliomielite e paralisia cerebral, apresentaram menor necessidade de prótese (18,18\%) quando comparados ao total de indivíduos para os 
quais esse tratamento foi indicado. Por fim, nenhum indivíduo apresentando anomalias congênitas foi encaminhado para confecção de prótese.

Com relação ao perfil comportamental dos 88 pacientes que necessitaram de próteses removíveis, todos foram atendidos em ambulatório, sete indivíduos (7,95\%) necessitaram de condicionamento psicológico e a maioria ( $89,77 \%)$ finalizou a reabilitação. A história odontológica prévia mostrou que a maioria $(71,59 \%)$ aceitou anestesia local. Quando questionados sobre a quantidade de medicações utilizadas, independentemente do grupo farmacológico, a maioria dos pacientes $(21,59 \%)$ utilizava mais de cinco tipos, seguida dos que ingeriam três medicações, e apenas 12 (13.64\%) não faziam uso de qualquer medicação.

Tabela 1 | Distribuição dos pacientes com necessidades especiais estudados, segundo o diagnóstico principal e a necessidade de prótese.

\begin{tabular}{c|c|c|c|c} 
Diagnóstico principal & Total & Percentual em relação ao total & PNE* encaminhados para prótese & Percentual em relação ao diagnóstico \\
\hline Alterações sistêmicas & 166 & $32,30 \%$ & 42 & 25,30 \\
\hline Deficiência física & 85 & $16,54 \%$ & 16 & 18,82 \\
\hline Distúrbios psiquiátricos & 45 & $8,75 \%$ & 10 & 22,22 \\
\hline Anomalias congênitas & 33 & $6,42 \%$ & 0 & 0 \\
\hline Total & 514 & $100 \%$ & 88 & \\
\hline
\end{tabular}

* PNE (Pacientes com Necessidades Especiais)

\section{DISCUSSÃO}

A baixa prevalência (17,11\%) desses PNE do CEOPoá encaminhados à reabilitação oral é um valor altamente contraditório se levarmos em conta que a literatura relata índices de perdas dentárias mais impactantes em $\mathrm{PNE}^{3}$. No Brasil, esse problema de saúde bucal é tão alarmante que, segundo o último levantamento oficial de saúde bucal, a média nacional dos indivíduos que necessitam de prótese foi de $68,8 \%$ entre os adultos (35 a 44 anos), mas muito maior (92,7\%) entre os idosos (65 a 74 anos) ${ }^{10}$. Mesmo em um país desenvolvido como o Japão, a prevalência de necessidade de prótese foi estimada em $54 \%^{11}$.

O achado deste estudo é muito importante, pois alerta para o problema sério da necessidade de adaptação de técnicas para solucionar o edentulismo entre PNE. O CEO-Poá provavelmente reflete a população de PNE no país, onde mesmo os indivíduos edêntulos e com necessidade de reabilitação oral foram considerados inaptos à sua execução e ao uso das próteses devido às diferentes limitações dessa população. Portanto, os fatores que influenciam a necessidade de próteses dessas pessoas foram avaliados neste estudo.

Quanto ao diagnóstico principal, os PNE com alterações sistêmicas foram os que mais necessitaram de próteses (25,3\%). Na literatura, artigos descrevem doenças variadas, incluindo hipertensão arterial e diabetes mellitus, seguidas de alterações cardiovasculares conduzindo ao estresse oxidativo, que desencadeia o estado hiperinflamatório, levando à periodontite e ao maior risco de edentulismo ${ }^{12,13}$. Essa pré-disposição torna-se mais evidente quando constatado que, após a melhora na saúde geral, muitos indivíduos apresentam diminuição na prevalência de cáries, doenças periodontais, e até de acometimentos peri-implantes ${ }^{14}$. Portanto, sugerimos que, apesar dos cuidados específicos durante o tratamento odontológico curativo, o perfil comportamental desse grupo provavelmente possibilitaria a execução e o 
uso de próteses . Esse grupo, por não apresentar alterações psicológico-comportamentais que prejudicassem a indicação de prótese, foi o grupo mais prevalente.

A segunda maior prevalência de necessidade de próteses foi encontrada entre os distúrbios psiquiátricos (22,2\%). Artigos associam perdas dentárias nesse grupo de pacientes ao abandono dos cuidados diários de higiene ${ }^{15}$. Porém, pesquisas também mostraram que alterações fisiológicas relacionadas à depressão, como o estado hiperinflamatório, podem levar à periodontite, com consequente mobilidade e perdas dentárias ${ }^{13}$.

Entretanto, os comportamentos depressivos, esquizofrênicos ou psicóticos podem melhorar com a recuperação tanto da estética oral quanto da mastigação e da fala. Porém, é necessário considerar que avaliamos um grupo com diferentes doenças, e que indivíduos com o mesmo diagnóstico podem apresentar comportamento e aceitação de tratamento distintos. Assim, os casos devem ser avaliados individualmente, podendo inclusive ser consideradas reabilitações complexas, como próteses fixas sobre implantes realizadas no âmbito hospitalar sob anestesia geral ${ }^{16}$.

Já nos deficientes físicos com sequelas de AVC, paralisia cerebral, entre outros, a baixa prevalência da necessidade de prótese $(18,18 \%)$ pode ser decorrente dos problemas motores. Nesse grupo de pacientes são comuns sintomas como rigidez muscular e movimentos involuntários nos lábios, língua e mandíbula, com consequentes alterações funcionais bucais. Essas disfunções limitam tanto a higiene bucal, levando a perdas dentárias, como o uso de próteses, uma vez que a musculatura oral se encontra alterada ${ }^{6}$.

No planejamento da reabilitação oral, o cirurgiãodentista precisará estar atento às características das sequelas orais de cada paciente para avaliar sua intensidade e ponderar se o paciente terá condições de colaborar em cada etapa da confecção da prótese, incluindo as moldagens, adaptações e os ajustes, e mantê-la após sua instalação. Em decorrência dessas limitações, neste estudo um pequeno percentual dos deficientes físicos foram aptos à confecção e uso de próteses removíveis, totais e parciais.

Como descrito anteriormente, a outra possibilidade seria a reabilitação com próteses fixas sobre implantes em âmbito hospitalar, porém esse serviço ainda não é disponibilizado na nossa instituição. Apesar de a literatura apontar bons prognósticos na reabilitação com implantes executados sob anestesia geral em PNE, fatores relevantes como higiene, presença de parafunções e qualidade óssea dependendo da localização e tamanho do edentulismo devem ser considerados ${ }^{17,18}$.

Os demais diagnósticos tiveram uma participação percentual menor. Isso se deve, principalmente, ao fato de que pacientes com acometimentos leves são atendidos nas Unidades Básicas de Saúde (UBS), encaminhados sem avaliação prévia por especialistas em PNE. Dos pacientes encaminhados para tratamento protético por especialistas em PNE, embora tenham sido atendidos no âmbito ambulatorial, todos apresentavam condições complexas, como uso de medicações múltiplas, intensidade das reações individuais ao tratamento e dificuldades na colaboração do núcleo familiar ou de cuidadores, as quais influenciam diretamente no sucesso da execução e na aceitação do uso das próteses removíveis.

Analisando a variável quantidade de medicação utilizada, independentemente do tipo de droga, a maior prevalência do uso de próteses ocorreu no grupo que ingeria cinco ou mais fármacos constantemente. Um estudo anterior mostrou que $55 \%$ dos pacientes com perdas dentárias ingeriam rotineiramente três ou mais fármacos ${ }^{15}$. Concordamos com esse estudo ao considerar que, independentemente do grupo ao qual as drogas pertençam, medicações diversas aumentam a probabilidade de o paciente diminuir o autocuidado 
e a higiene bucal, impactando na incidência de cáries e doenças periodontais, perda dentária e, consequentemente, na necessidade do uso de prótese.

O uso de vários fármacos concomitantemente também predispõe à xerostomia, agravando periodontites e outras doenças bucais ${ }^{15}$. As parafunções são outra causa significativa das perdas dentárias e que pode estar associada ao uso de medicações, embora essa associação não tenha sido investigada. Por exemplo, existem evidências de associação entre o bruxismo e os estimulantes do sistema nervoso central, muito utilizados em casos de transtornos de déficit de atenção e hiperatividade (TDAH), assim como os inibidores seletivos da receptação de serotonina (ISRS), comumente utilizado por pacientes depressivos ${ }^{16}$.

No nosso estudo, entre os PNE selecionados para tratamento protético prevaleceu o gênero feminino, o que pode decorrer do fato de que, na população em geral, o autocuidado é maior entre as mulheres, fazendo que a procura por próteses seja maior entre essas pacientes ${ }^{17}$. A necessidade de prótese nos PNE foi diretamente proporcional ao aumento da idade, como ocorre na população em geral. Os idosos brasileiros, além de mais propensos às doenças dentárias, podem ter sido submetidos à odontologia que preconizava procedimentos na sua maioria mutiladores ${ }^{18}$. Outros estudos também associaram o envelhecimento às perdas dentárias nos PNE, e sugeriram como solução tratamentos odontológicos curativos prévios em deficientes jovens ${ }^{19}$.

Este estudo fez uma análise retrospectiva de pacientes atendidos em um Centro de Especialidades Odontológicas municipal e, portanto, teve limites quanto à representatividade nacional. Além disso, como o objetivo do estudo foi caracterizar a necessidade de prótese removível em pacientes portadores de necessidades especiais, a faixa etária foi ampla, bem como o número de diagnósticos, o que limitou o aprofundamento da avaliação. Porém, consideramos esses dados importantes para o planejamento do atendimento de saúde.

Um fator limitador do estudo foi que indivíduos com acometimentos leves atendidos nas UBS foram direcionados à reabilitação protética no CEO sem a avaliação de especialistas em PNE e, portanto, não foram incluídos na nossa amostra. Por outro lado, um aspecto importante deste estudo foi mostrar que na maioria dos casos a reabilitação oral proposta foi finalizada (89,77\%).

Sendo esse serviço público e independente dos recursos financeiros do paciente, os tratamentos não finalizados foram preponderantemente decorrentes de óbito ou mudança de município. Esse fator reforça a importância da instalação de novos CEO, para que os especialistas em PNE estejam próximos à moradia dessa população ${ }^{20}$. Outro diferencial para o estudo foi a disponibilização de transporte pelo Centro para os PNE, otimizando o comparecimento às consultas.

A quantidade exorbitante de pessoas com deficiência na população mundial está além da compreensão da maioria das pessoas. A própria variedade de doenças e de nível de acometimento dificultam os estudos e divulgação de informações ${ }^{21}$. Recentemente foi relatado que a Organização Mundial da Saúde uniu-se a universidades canadenses e americanas, planejando divulgar números sobre as necessidades odontológicas dos PNE e capacitar cirurgiões-dentistas para atender essa demanda. O desafio desse programa é sensibilizar órgãos legislativos e provedores de saúde ${ }^{22}$.

Os fatores influenciadores nas soluções protéticas dos PNE foram analisados e discutidos para que esse problema grave na saúde pública e privada seja amenizado. Este estudo poderá nortear a implementação de projetos tanto no setor privado como no público, levando em consideração o diagnóstico, a idade e o comportamento do públicoalvo. As características dessa população podem orientar a aquisição de equipamentos e materiais odontológicos, assim como na adaptação do 
âmbito de atendimento. Porém, novas pesquisas são necessárias para melhor delimitar o perfil de cada grupo dos PNE, em uma população maior e mais específica de pacientes, e assim identificar com maior clareza as intervenções que são eficazes para a reabilitação oral e, consequentemente, para a qualidade de vida dessa população.

\section{CONCLUSÕES}

Neste estudo, as variáveis influenciadoras no uso de prótese em PNE foram gênero, idade, diagnóstico principal e quantidade de medicação utilizada usualmente, independentemente do grupo ao qual as drogas pertençam, pois os que utilizavam mais de cinco medicações usualmente apresentaram maior necessidade do uso de prótese. Como na população em geral, gênero e idade também influenciaram, sendo prevalente a necessidade de prótese entre mulheres e idosos. $\mathrm{O}$ fator mais evidente foi o diagnóstico principal, sendo que a predominância foi entre os PNE incluídos na categoria das alterações sistêmicas, seguidos dos deficientes físicos e portadores de distúrbios psiquiátricos.

\section{REFERÊNCIAS}

1. Haag DG, Peres KG, Balasubramanian M, Brennan DS. Oral conditions and health-related quality of life: a systematic review. J Dent Res. 2017 Jul;96(8):864-74. doi/10.1177/0022034517709737.

2. Friedman PK, Lamster IB. Tooth loss as a predictor of shortened longevity: exploring the hypothesis. Periodontol 2000. 2016 Oct;72(1):142-52. doi: 10.1111/prd.12128.

3. Corcuera-Flores JR, Delgado-Muñoz JM, Ruiz-Villandiego JC, Maura-Solivellas I, Machuca-Portillo G. Dental treatment for handicapped patients: sedation vs general anesthesia and update of dental treatment in patients with different diseases. Med Oral Patol Oral y Cir Bucal. 2014 Mar;19(2):e170-6. doi:10.4317/medoral.19555.

4. Conselho Federal de Odontologia (Brasil). Resolução n. 22, de 27 de dezembro de 2001. Normas sobre anúncio e exercício das especialidades odontológicas e sobre cursos de especialização. Diário Oficial da União; 25 jan 2002.
5. Brown LF, Ford PJ, Symons AL. Periodontal disease and the special needs patient. Periodontol 2000. 2017 Jun;74(1):18293. doi: 10.1111/prd.12198.

6. Ribeiro GR, Campos CH, Rodrigues Garcia RCM. Influence of a removable prosthesis on oral health-related quality of life and mastication in elders with Parkinson disease. J Prosthet Dent. 2017 Nov;118(5):637-42. doi: 10.1016/j.prosdent.2016.12.018.

7. Al-Imam H, Özhayat EB, Benetti AR, Pedersen AM, Gotfredsen K. Oral health-related quality of life and complications after treatment with partial removable dental prosthesis. J Oral Rehabil. 2016 Jan;43(1):23-30. doi: 10.1111/joor.12338.

8. Kattadiyil MT, AlHelal A, Goodacre BJ. Clinical complications and quality assessments with computer-engineered complete dentures: a systematic review. J Prosthet Dent. 2017 Jun;117(6):721-8. doi: 10.1016/j.prosdent.2016.12.006.

9. Haddad AS. Odontologia para pacientes com necessidades especiais. São Paulo: Santos; 2007.

10. Brasil. Ministério da Saúde. Secretaria de Atenção à Saúde. Secretaria de Vigilância em Saúde. SB Brasil 2010: Pesquisa Nacional de Saúde Bucal: resultados principais. Brasília, DF: Ministério da Saúde; 2012.

11. Matsuyama Y, Aida J, Takeuchi K, Tsakos G, Watt RG, Kondo $\mathrm{K}$, et al. Inequalities of dental prosthesis use under universal healthcare insurance. Community Dent Oral Epidemiol. 2014 Apr;42(2):122-8. doi: 10.1111/cdoe.12074.

12. Kumar J, Teoh SL, Das S, Mahakknaukrauh P. Oxidative stress in oral diseases: understanding its relation with other systemic diseases. Front Physiol. 2017 Sep;8:1-15. doi: 10.3389/fphys.2017.00693.

13. Chapple IL, Bouchard P, Cagetti MG, Campus G, Carra MC, Cocco F, et al. Interaction of lifestyle, behaviour or systemic diseases with dental caries and periodontal diseases: consensus report of group 2 of the joint EFP/ORCA workshop on the boundaries between caries and periodontal diseases. J Clin Periodontol. 2017 Mar;44(Suppl 18):S39-51.

14. Nobre MA, Maló P. Prevalence of periodontitis, dental caries, and peri-implant pathology and their relation with systemic status and smoking habits: results of an open-cohort study with 22009 patients in a private rehabilitation center. J Dent. 2017 Dec;67:36-42. doi: 10.1016/j. jdent.2017.07.013.

15. Latif TM, Vieira AR. Risk factors and comorbidities associated with complete edentulism in individuals younger than fifty years of age. J Dent Oral Heal. 2017 Aug;4:1-6. 
16. Drisdale III JK, Thornhill MG, Vieira AR. Specific central nervous system medications are associated with temporomandibular joint symptoms. Int J Dent. 2017;2017:1-5. doi: 10.1155/2017/1026834.

17. Zusman SP, Ponizovsky AM, Dekel D, Masarwa A, Ramon T, Natapov L, et al. An assessment of the dental health of chronic institutionalized patients with psychiatric disease in Israel. Spec Care Dent. 2010 Jan-Feb;30(1):18-22. doi: 10.1111/j.1754-4505.2009.00118.x.

18. Kim I, Kuk TS, Park SY, Choi Y, Kim HJ, Seo K. Prognosis following dental implant treatment under general anesthesia in patients with special needs. J Dent Anesth Pain Med. 2017 Sep;17(3):205-13. doi: 10.17245/jdapm.2017.17.3.205.

19. Romero-Pérez M, Mang-de la Rosa M, Lopez-Jimenez J, Fernandez-Feijoo J, Cutando-Soriano A. Implants in disabled patients: a review and update. Med Oral Patol Oral Cir Bucal. 2014 Sep;19(5):e478-82. doi: 10.4317/medoral.19564.

20. Azevedo JS, Azevedo MS, Oliveira LJC, Correa MB, Demarco FF. Uso e necessidade de prótese dentária em idosos brasileiros segundo a Pesquisa Nacional de Saúde Bucal (SBBrasil 2010): prevalências e fatores associados. Cad Saude Publica. 2017;33(8):1-12. doi: 10.1590/0102-311Xoo054016.

21. Morgan JP, Minihan PM, Stark PC, Finkelman MD, Yantsides KE, Park A, et al. The oral health status of 4,732 adults with intellectual and developmental disabilities. J Am Dent Assoc. 2012 Aug;143(8):838-46.

22. Waldman HB, Wong A, Perlman SP. Comprehending the number of individuals with disabilities and the need for oral health services. J Clin Pediatr Dent. 2017 Mar;41(2):83-6. doi: 10.17796/1053-4628-41.2.83. 